\title{
Design of a Telerobotic Interface System by Using Object Recognition Techniques
}

\author{
Raül Marín ${ }^{1}$, Pedro José Sanz ${ }^{1}$, Antonio José Jimeno ${ }^{1}$, and Jose Manuel Iñesta ${ }^{2}$ \\ ${ }^{1}$ Dept. of Computer Science, "Jaume I" University, Riu Sec Campus, Castellón, Spain \\ \{rmarin, sanzp\}@inf.uji.es \\ ${ }^{2}$ Dept. of Computer Science, Alicante University, Alicante, Spain
}

\begin{abstract}
This paper presents a real application that adds learning capabilities to a telerobotic system, designed formerly to manipulate everyday objects over a board. The user interaction is based on a restricted natural language and object recognition techniques have been designed specifically in order to acquire a system that responds as fast as possible to the user commands. First of all, the article will introduce the overall description of the system, in order to get a general idea of its main functions. Then, it will focus on a comparison of different recognition procedures that have been already added to the implementation. It will show the algorithm that fits better to the real needs of the project, looking for a balance between efficiency and recognition capabilities.
\end{abstract}

\section{Introduction}

In the recent years telerobotic systems have become more and more important, particularly due to the fact that the number of inaccessible and risky work-sites has increased. Telerobotic systems are now evolving beyond the function of being pure extensions of their operators, and nowadays this is one of the major concerns for researchers in the field. According to [6], a good telerobotic system should be both transparent and semi-autonomous. The system should be designed to augment the operator's capabilities by semi-automating tasks whenever it is possible and costeffective.

In this paper we present a real application whose goal is to design and implement a telerobotic system that includes learning capabilities and aims to a Human-Robot communication based on a subset of the natural language. Besides this, the paper is focused on the analysis of several recognition procedures in order to realise which one of them presents a higher percentage of hits and a better performance, allowing the interface to be used in a real time manner. The system, which provides control over a generic vision-guided robot, follows an Object Oriented Distributed Arquitecture by means of the CORBA standard [12]. This allows the system to interconnect multiple modules running on different platforms and to be implemented using different programming languages (PROLOG, LISP, C++, Java, etc). Besides this, the user inter- 
face is defined by using the Java programming language, allowing it to be run on multiple platforms or even better, over the Internet.

\section{Related Work}

Many different telerobotic systems have been reported since Goertz introduced the first teleoperator at the Argonne National Laboratory four decades ago [4]. As expected, most of the former systems required expensive hardware and software in order to implement a user interface that operates far away from the robot. Nowadays, the expansion of the World Wide Web has allowed a increasing number of user interfaces that control remote devices not only for robots, but also cameras, coffee pots, and cake machines, to name some. The first telerobotic systems with this kind of interface were presented by researchers from the University of Southern California (USC) and the University of Western Australia (UWA). The Mercury Project [5], carried out at the USC, led to the development of a system in which the manipulator was a robotic arm equipped with a camera and a compressed air jet, and the interface consisted of web page that could be accessed using any standard browser. The telerobotic system developed at the UWA [15] lets the user control an industrial robot to manipulate objects distributed on a table. The user interface allows an operator to specify the coordinates of the desired position of the arm, the opening of its gripper and other multiple parameters.

In our system, we aim to allow operators to use their voice to control the robot movements. Besides this, the system is able to learn from experience and the user interaction. Thus, it will accept high-level commands such as "pick up the small pen", that require the use of some acknowledge to be carried out. On the other hand, as we will see in the following sections, the recognition algorithm has been selected in order to allow it to be used as a Real-Time application. The Robot response must come up in less than a few seconds. In fact, the problem to control a robot via the web will have many additional difficulties because it is a very uncertain environment where the velocity of data transmission can not be guaranteed, and the delay is always present. A very recent discussion about this topics in the telerobotics domain can be found in [3]. However, we have selected the web as the best way of communication between the user interface and the robot because it allows the manipulator to be accessed from any computer connected to the internet. It makes this connection cheap and easy, and we consider these reasons are sufficient to prefer the Internet to a dedicated network that would allow us a much better performance. Besides, as new broadband Internet connections are coming up (fe. ADSL or Satellite) this web bottleneck will fade out too.

\section{Overall System Description}

This section summarises the complete project we are involved on, which consists of a telerobotic system based on Computer Vision and a static robot, programmed in real time by a remote interface using a restricted natural language input. 
A camera is used to obtain the environment images with the objects the robot can access to. These images are transferred to the computer running the user interface, using Internet as the access medium, and then showed to the user in order to allow him to know the real environment state. The restricted natural language input gets into the system via the user interface, which translates in real time these commands into another language that the robot can understand.

First of all, we should address the existence of a previous work explained in [13], where all the problems related to grasping and camera operations are already sorted out. With the aim to make good use of the features already implemented there, we have designed a Server application that offers low level grasping and camera services. We will refer to this subsystem as "Robot Server". The Robot Server is in charge of setting up the robot and the camera, and controlling them. This subsystem runs on a PC and interacts with a robot SCARA via the parallel port. The Robot Server capabilities are used remotely by the user interface running on the Internet through a distributed object oriented interface implemented with CORBA (Common Object Request Broker Arquitecture) [12].

Secondly, we will introduce the user interface capabilities. It gets an image as input with the objects in the scene, a Database with descriptions of the objects that the system can recognise and a command specified in the restricted natural language by the user. The output would be a low-level set of commands in a protocol that the Robot Server can understand. We will refer to this protocol as GCCP (Grasping and Camera Control Protocol). This GCCP set of commands will be evaluated first by the Robot Server and finally translated to a different set of low level commands depending on the particular robot we are interacting with. All this process is summarised in fig. 1.

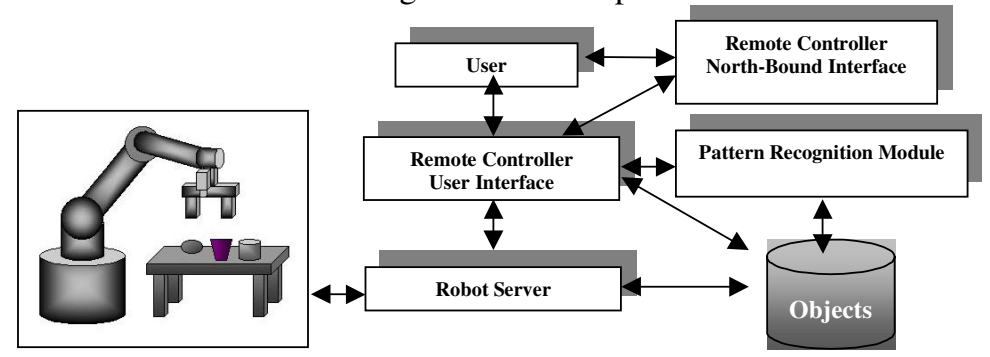

Fig. 1. Overall System Arquitecture. This figure is a brief description of the different entities belonging to the system. The Robot Server knows about GCCP, the particular robot interacting with and grasping characterisation. User interface recognises a few voice commands and translates them to GCCP by making use of the objects database. Acknowledge retrieved from user interaction is updated to the Database (learning capability). 


\section{User Interface Description}

Basically, the user interface consists of a Java application that allows users to obtain images of the robot's workspace and configuration information about the robot and the camera, send commands to the Robot Server for controlling the robot and access the object database in the Database Server to read and update the robot acknowledge.

The user interface, which is shown in fig. 2, allows the use of the restricted natural language for specifying the commands to be sent to the Robot Server. This means users can employ the same names and adjectives they use to refer to objects in the real world, as well as the same constructions for expressing location (above, at the left hand of, etc). The natural language module translates user commands into GCCP interactions that the robot can understand. This module has access to the database of known objects in order to check if the objects that users are referring to are or not manageable by the system. When an object cannot be recognised by the module described in the previous section the user will be asked for the object name.

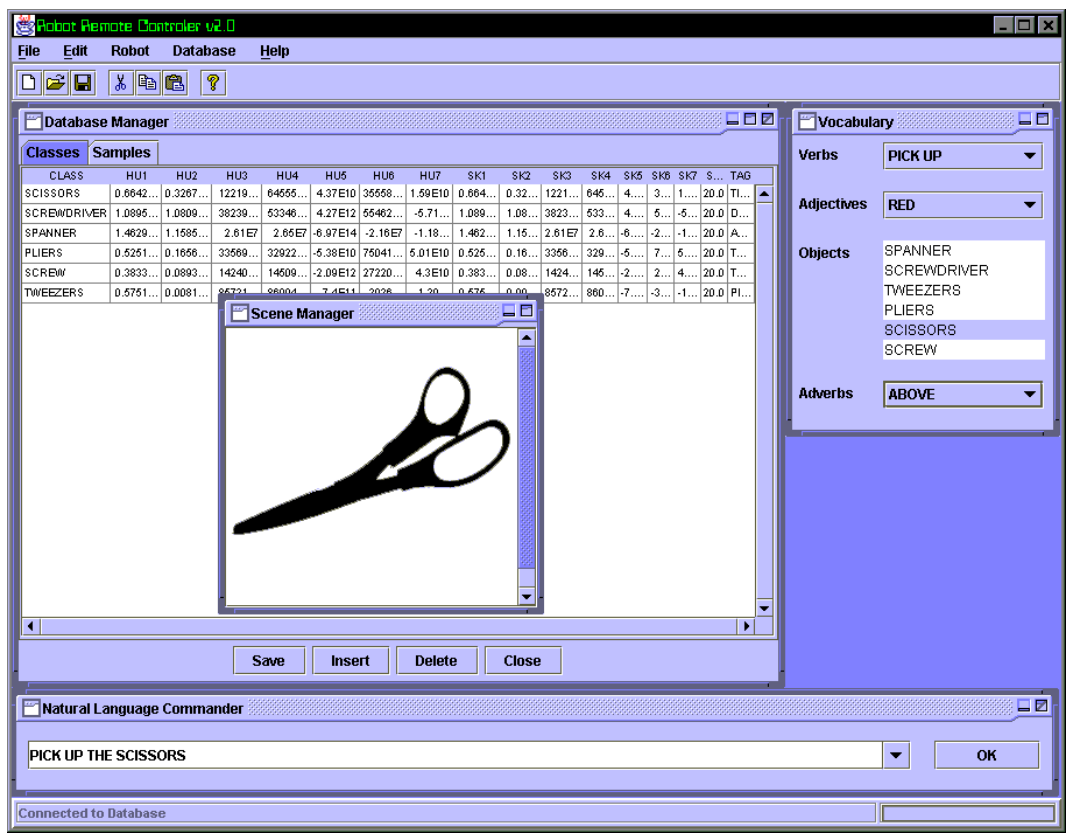

Fig. 2. User interface (remote controller) 


\section{Object Recognition Analysis}

As the system is intended to be used in a real-time manner, this section analyses several recognition algorithms that may allow this kind of interaction. The object recognition and learning tasks are performed by a user interface module, which performs an analysis of the real images received from the Robot Server or provided by the user, and then computes a set of descriptors that identify uniquely the objects contained in the different scenes. These descriptors, known as "HU descriptors", are based on the invariant-moments theory [7]. Once we compute the "HU descriptors" for a given object sample, we apply the learning recognition procedure to know if this object belongs to an already learned class or whether it identifies a category that must be added to the robot acknowledge set.

Before the classification process and the $\mathrm{HU}$ descriptors extraction of an object the image is preprocessed and segmented in order to identify the objects belonging to the scene. This action is accomplished through a previous binarysation of the scene and then a simple segmentation algorithm that searches from top to bottom and from left to right the different objects to be treated. The idea is to make it simple enough in order to obtain as much performance as possible.

In order to select the best algorithm that fits our needs, we have implemented several distances and learning recognition algorithms, and then we have extracted different timing and quality recognition results that are explained in the next section. We will be able then to select the best set of algorithms needed for our current necessities.

\section{Distances}

The distances selected to perform the comparative analysis have been "Euclidean distance", "Normalised distance", "Extended Euclidean distance"[10] and "Per-class Extended Euclidean distance". The last three follow the same philosophy that is applying a weighted Euclidean distance to the object samples (see formulaes (1), (2) and (3)). The only difference between them is the definition of these weights. Note "n" as the number of elements that define a HU descriptor, "wi" as the weight applied to an element of a HU descriptor, and " $\mathrm{i}$ " as the integer varying between 1 to " $\mathrm{n}$ " that identifies a component of the HU descriptor array.

$$
d(X, Y)=\sqrt{\sum_{i=1}^{n} w_{i}\left(X_{i}-Y_{i}\right)^{2}}
$$

Formulae (1). Generic distance between vectors X and Y. Depending on the distance algorithm applied, the weights will vary accordingly. 


$$
m_{i}=\frac{1}{n} \sum_{k=1}^{n} x_{k i}
$$

Formulae (2). Average between the samples belonging to a class for the "i" descriptor.

$$
s_{i}=\frac{1}{n-1} \cdot\left(\sum_{k=1}^{n} x_{k i}^{2}-m_{i}\right)
$$

Formulae (3). Deviation of the samples belonging to a class for the i descriptor.

As we can see in Formulae (4), the "Normalised distance" uses as weights the inverse of the variance for every class. It means we must manage and update at any time the per-class variance in order to apply this distance. But the point is: "The weights are different for each class" so, its cost will be higher.

$$
w_{k i}=\frac{1}{s_{k i}}
$$

Formulae (4). Normalized distance weight definition. As more stability presents a HU descriptor column, more significant will it be for the recognition criteria. The weights are different for each one of the classes. Note " $k$ " as identifying the specific class and "i" as the column belonging to the HU descriptors array.

On the other hand, we designed the "Extended Euclidean distance" in order to speed up the "Normalised distance" (formulae (5)). It is based on a previous statistical study that defines the constant weights that will be used by this distance. The important thing is that the weights to be applied are known in advance and we do not have to manage any per-class variance in order to implement the recognition procedure. Besides this, as explained in [10], in order to perform this previous statistical study, we must know in advance the set of classes that will be entered into the system. Basically, it is designed for closed environments where the classes are well known in advance.

$$
w_{i}=\left(\sum_{k=1}^{N}\left(\frac{1}{s_{k i}}\right)\right) \cdot\left(\sum_{k=1}^{N}\left(\frac{1}{w_{k i}}\right)\right)
$$

Formulae (5). Extended Euclidean distance definition. This takes into account the negative effect that have the biggest $\mathrm{HU}$ components into the recognition process. Thus, it extends the Normalised approach in order to benefit the smaller HU components. Besides this, as the objective is to have a constant weight for the whole recog- 
nition algorithm, the formulae represented above is applied. Note " $\mathrm{N}$ " as the number of classes.

And finally, in order to include the "Extended Euclidean distance" capabilities to an open system where the classes are not well known in advance, we have designed the "Per-class Extended Euclidean distance" (see Formulae (6)). Basically it is a mixture of the "Extended Euclidean distance" and the "Normalised" one. First of all, it defines different weights for each class into the acknowledge base (as Normalised does), and finally, it takes into account scaling properties of each HU descriptor as the "Extended Euclidean distance" does define.

$$
w_{k i}=\frac{1}{s_{k i}} \cdot \frac{1}{m_{k i}}
$$

Formulae (6). Per-class Extended Euclidean Distance definition. The weights to be applied are different for each on of the classes. It takes into account the component scale in order to perform the recognition.

\section{Learning Algorithms}

In order to perform the comparative of different recognition algorithms we have selected two of them, "Minimum Distance Classifier" and "K-Nearest Neighbors Classifier", and then we made an extension to their definition in order to give them learning capabilities. Basically, when a sample is not recognised as belonging to any class of the current acknowledge base we ask the user for a new class name and add it to the classes database. Once this happens the system is able to recognise samples belonging to that class, and that means the program has learned a new class definition. On the other hand, when a sample is conceived as belonging to an already known class, the class definition is refined too. For the "Minimum Distance Recognition" it is accomplished by maintaining into the class definition the average of the its whole sample set. This means, if a new sample belongs to the class, the average must be updated accordingly. Besides this, for the "K-Nearest neighbors", we have to add the new sample definition to the acknowledge database as it is.

\section{Results}

The main objective of the present study is selecting the best combination of distance and recognition algorithm that accomplishes the process as fast as possible and with an acceptable quality. To do so a battery test has been designed by taking 120 images belonging to 6 different kinds of objects. We can see a sample of each class in fig. 3 . 


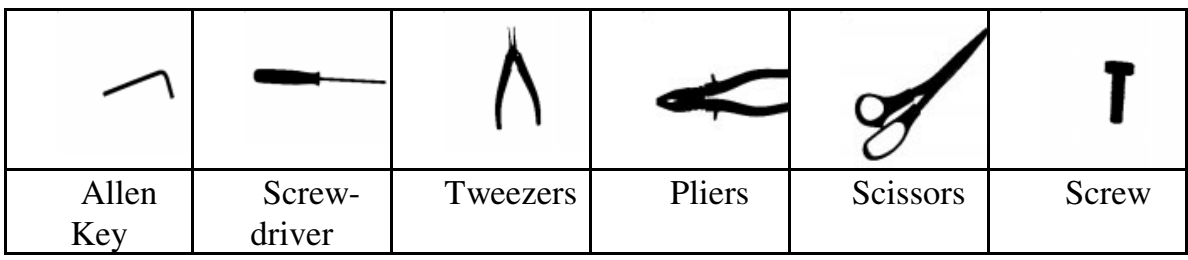

Fig. 3. Classes of objects for the battery test.

As we can see in fig. 4 , we have represented the 8 combinations between distances (Euclidean, Modified, Normalised and Per-Class) and classifiers (Minimum Distance and K-nearest neighbors). For every one of these combinations we launch the procedure to classify the 120 samples. The result is the time in seconds expended to classify the 120 samples and the \% of samples recognised properly.

Besides this, we consider the best algorithm as the one that maximises the $\%$ of samples recognised properly divided by the time spent to do so. This relationship can be observed in fig. 5 , in graphical manner.

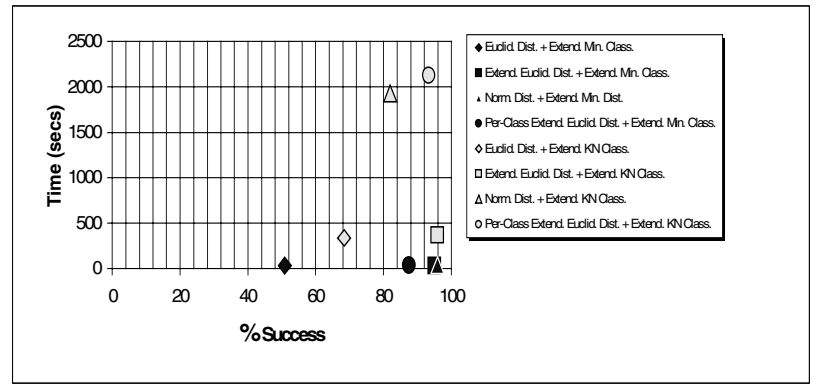

Fig. 4. Success X Time Results. For every algorithm it represents the \% Success and the Time that expended to recognise the 120 samples.

As we can see in fig. 4 the algorithm to apply seems to be the "Extended Euclidean Distance" with the "Minimum Distance Classifier". It performs very fast and offers a $\%$ of success over the $95 \%$. Besides this, we introduced in the section above that the "Extended Euclidean Distance" needs to have a previous statistical analysis in order to calculate the constant weights that work better. This kind of distance has been designed for systems where the classes managed are always the same. If a new class has to be added to the acknowledge database the statistical process should be carried out again.

For open systems, where the number of classes to be managed is not limited, we should select the next algorithm. It is the one composed by the "Normalised Distance" and the "Minimum Distance Classifier". 


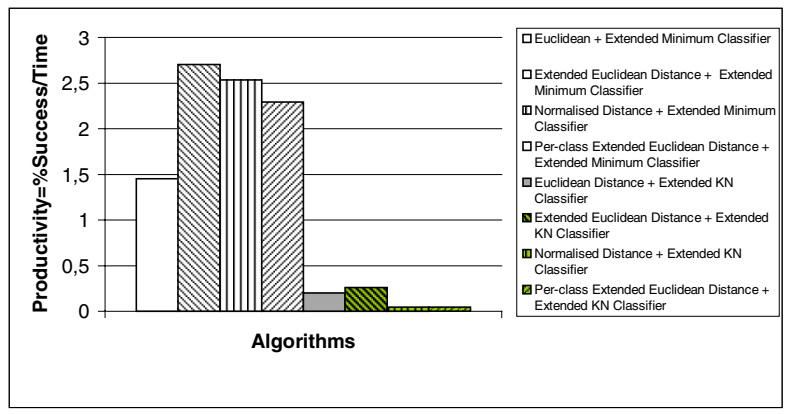

Fig. 5. Productivity Results. For every algorithm it represents the \% Success/Time. The best algorithm will be the one that is able to recognise properly more samples in a little amount of time.

\section{Conclusions and Future Work}

The conclusions of the present study are both, using the "Extended Euclidean Distance" for close systems (always managing the same classes) and using the "Normalised Euclidean Distance" for open systems. Both of them are to be used with the "Extended Minimum Distance Classifiers" that provides learning capabilities to the traditional "Minimum Distance Classifier". The "K-nearest neighbors Classifier" may not be used because it is computationally too expensive and would make impossible the real-time approach required to our telerobotic system.

Future steps in the project will be oriented towards completing and improving the set of services that each component of the system must provide. In the user interface, two main points to improve are, in order to allow a more natural interaction with the user, the support for voice command input and the richness of the language the system can understand. We also plan to implement task learning capabilities, so that the user can define tasks as a sequence of other already-known ones. Besides this, the next version of the user interface will allow the user to manipulate the objects on the scene graphically by simulating the results before sending the command to the Remote Server. It means the user will be able to control the robot using both, natural language and mouse interaction.

As a final conclusion, we believe that in the years to come more intelligence is expected to be added to both the robot and the interface sides of the system, making user-robot interaction flow in a more natural way.

\section{Acknowledgements}

This paper describes research done at the Robotic Intelligence Laboratory, Jaume-I University. Support for this laboratory is provided in part by Generalitat Valenciana 
under project GV97-TI-05-8, by CICYT under TAP98-0450, and by Fundació CaixaCastelló under P1B97-06.

\section{References}

[1] Baldwin, J., Basu, A., Zhang, H. (1998): Predictive Windows for Delay Compensation in Telepresence Applications: Proceedings of the IEEE Conference on Robotics and Automation: Leuven, Belgium, 1998.

[2] Everett, S.E., Isoda, Y., Dubey, R. V. (1999): Vision-Based End-Effector Alignment Assistance for Teleoperation: Proceedings of the IEEE Conference on Robotics and Automation: Detroit, Michigan, 1999.

[3] Fitzpatrick. Live Remote Control of a Robot via the Internet. IEEE Robotics and Automation Magazine Vol. 6, No 3, pp.7-8. 1999.

[4] Goertz, R. and Thompson, R. (1954): Electronically Controlled Manipulator: Nucleonics, 1954.

[5] Goldberg, K. (1995): Desktop Teleoperation via the World Wide Web: Proceedings of the IEEE Conference on Robotics and Automation: Nagoya, Japan, 1995.

[6] Hopper, D., Boddy, C., Reilly, D. (1995): Human Centered vs Task-Centered Design of Telerobotic Systems: Human-Oriented Design of Advanced Robotics Systems, postprint volume from the IFAC Workshop, Viena, 1995.

[7] Hu, M.K. (1962): Visual Pattern Recognition by Moment Invariants: IRE Transactions on Information Theory, vol. IT-8, pp. 179-187, 1962.

[8] Jägersand, M. (1999): Image Based Predictive Display for Tele-Manipulation: Proceedings of the IEEE Conference on Robotics and Automation: Detroit, Michigan, 1999.

[9] Sun Microsystems (1999): Java Speech API Home Page:http://java.sun.com/products/java-media/speech/

[10] Marín, R., Recatalá, G., Sanz, P.J., Iñesta, J.M, del Pobil, A.P. (1998): Distributed Arquitecture for a Learning Telerobotic System: Seventh European Workshop on Learning Robots (EWLR-98), Edinburgh, 1998.

[11] Ogasawara, T., Hirukawa, H., Kitagaki, K., Onda, H. Nakamura, A., Tsukune, H. (1998): A Telerobotics System for Maintenance Tasks Integrating Planning Functions Based on Manipulation Skills: Proceedings of the IEEE Conference on Robotics and Automation: Leuven, Belgium, 1998.

[12] OMG (1999): The Common Object Request Broker, Aquitecture and Specification (CORBA): http://www.omg.org/corba/cichpter.htm

[13] Sanz PJ, del Pobil AP, Iñesta JM, Recatalá G. "Vision-Guided Grasping of Unknown Objects for Service Robots". In Proc. IEEE Intl. Conf. on Robotics and Automation, 3018-3025, Leuven, Bélgica, 1998.

[14] Simmons, R.G. (1997): Xavier, experience with a Layered Robot Arquitecture: ACM magazine Intelligence, 1997.

[15] Taylor, K., Trevelyan, J. (1995): Australia's Telerobot on the Web: Proceedings of the $26^{\text {th }}$ International Symposium on Industrial Robots, Singapure, 1995. 